\title{
EFECTO DE DOS ESPECIES DE ÁRBOLES REMANENTES \\ Y DE UN PASTO EN LA CAPACIDAD AMORTIGUADORA DEL pH
} DEL SUELO EN UN ECOSISTEMA TROPICAL ESTACIONAL

\author{
MARIBEL NAVA-MENDOZA, LEOPOLDO GALICIA y FELIPE GARCíA-OLIVA \\ Instituto de Ecología, UNAM, AP 27-3 Xangari, 58089 Morelia, Michoacán, México.
}

\begin{abstract}
Resumen: La capacidad amortiguadora del $\mathrm{pH}$ del suelo fue afectada por dos especies de árboles remanentes de la selva y por una especie de pasto. La diferencia más importante entre las dos especies de árboles remanentes fue el grado de neutralización del complejo de intercambio catiónico: el suelo de C. elaeagnoides estuvo dominado por los cationes alcalinos (i.e. $\mathrm{Ca}^{2+}, \mathrm{Mg}^{2+}, \mathrm{K}^{+}$) a diferencia del suelo de C. eriostachys, que estuvo dominado por $\mathrm{H}^{\prime}$, debido a su mayor tasa de nitrificación. En contraste, la especie de pasto tuvo una menor capacidad de amortiguamiento del pH y el factor principal que controló el pH en la solución del suelo fue la fuerza del ácido. Esto sugiere, que las condiciones de manejo en este ecosistema han reducidos los mecanismos de protección de nutrientes del suelo, asociados principalmente a las especies vegetales.

Palabras clave: Caesalpinia erioslachys Benth., Cordia elaeagnoides De., Panicum maximum Jacq., pradera, nitrificación

Abstract: Soil pH buffer capacity was affected by the two forest remnant tree species and one pasture species. The differences in the degree of neutraJization of the soil exchange cornplex was the main difference between the two rernnant tree species: base cations (i.e. $\mathrm{Ca}^{2+}, \mathrm{Mg}^{2+}, \mathrm{K}^{+}$) occupied the exchange complex in the soil associated to C. elaeagnoides, in contrast to that associated to C. eriostachys, because it was occupied by $\mathrm{H}^{+}$due to high nitrification rates. The pasture species has the lower $\mathrm{pH}$ buffer capacity and the acid strength was the main factor which controlled $\mathrm{pH}$ of soil solution. These results suggest that soil nutrient protection mechanisms were reduced by the pasture management, mainly associated to plant species.

Key words: Caesalpinia eriostachys Benth., Cordia elaeagnoides DC., Panicum maximum Jacq., pasture, nitrification.
\end{abstract}

A pesar de que desde hace tiempo la vegetación ha sido identificada como un factor fundamental en la formación del suelo (jenny, 1941), se ha considerado tradicionalmente que las características del mismo son independientes de las especies vegetales que sustentan. Un ejemplo de esto ha sido el $\mathrm{pH}$; su dinámica ha sido explicada sólo en función de sus componentes inorgánicos (i.e. la composición mineralógica; Buckman y Brady, 1970). Actualmente, se han desarrollado varios trabajos que reportan que las especies vegetales juegan un papel muy importante en el comportamiento del $\mathrm{pH}$ del suelo (Binkley y Sollins, 1990; Rhoades y Binkley, 1996; Finzi et al., 1998). El estudio de los factores que controlan el $\mathrm{pH}$ del suelo es fundamental, ya que determina la disponibilidad de los nutrientes del suelo y la solubilización de elementos tóxicos (Rusell, 1973; Buckman y Brady, 1970), así como la presencia de diferentes grupos microbianos (Buckman y Brady, 1970).

Entre los mecanismos que explican el efecto de las especies vegetales en la acidez del suelo, encontramos: la absorción de cationes y aniones intercambiables (Alban, 1982), la nitrificación incrementada por la fijación de nitrógeno (van Miegroet y Cale, 1984) y la producción de hojarasca rica en ácidos orgánicos (Howard et al., 1998). Binkley y colaboradores (1989) proponen que el $\mathrm{pH}$ de la solución del suelo puede estar determinado por: 1] la cantidad de ácidos, siendo la actividad biológica la principal fuente de $\mathrm{H}^{+}$, 2] el grado de disociación de los ácidos, donde la saturación de bases juega un papel muy importante y 3] la fuerza de los ácidos, que está relacionada con su afinidad a las superficies de intercambio catiónica $\left(\mathrm{pK}_{\mathrm{a}}\right)$. 
El estudio del efecto de las especies vegetales sobre la acidez del suelo se ha centrado en la comparación de especies fijadoras de nitrógeno con especies no fijadoras, principalmente en plantaciones forestales (Binkley y Sollins, 1990; Rhoades y Binkley, 1996). La hipótesis que sustenta esta comparación ha sido que la incorporación de $\mathrm{N}$ por las especies fijadoras, incrementa la producción de $\mathrm{H}^{+}$libres debido a la nitrificación, los cuales desplazan a los catiónicos básicos $\left(\mathrm{Ca}^{2+}, \mathrm{Mg}^{2+}, \mathrm{K}^{+}\right)$del complejo de intercambio, generando una reducción del $\mathrm{pH}$ del suelo (van Miegroet y Cole, 1984). La presencia de especies vegetales que incrementan la disponibilidad de $\mathrm{N}$ en el suelo, favorece la acidificación del mismo, siempre y cuando exista una alta tasa de lixiviación de nitratos.

El presente trabajo tiene como objetivos estudiar: 1] el efecto de dos especies de árboles remanentes de la selva en el $\mathrm{pH}$ del suelo bajo condiciones de pradera, una de los cuales incorpora mayor $\mathrm{N}$ disponible al suelo (C. eriostachys; Dockersmith et al., 1999; L. Galicia, com. pers.) y 2] comparar el efecto de estas dos especies con una especie de pasto de la pradera, que ha estado sujeta a manejo continuo en un ecosistema tropical estacional en la costa de Jalisco.

\section{Materiales y métodos}

Sitio de estudio. Las muestras de suelo fueron recolectadas en el ejido de San Mateo, municipio La Huerta en el estado de Jalisco $\left(19^{\circ} 29^{\prime} \mathrm{N}\right.$ y $\left.105^{\circ} 01^{\prime} \mathrm{O}\right)$. $\mathrm{El}$ relieve se caracteriza por ser lomeríos con pendientes convexas (Rodríguez, 1999). El tipo de suelo dominante es Entisol (USDA; Solís, 1993), sobre rocas riolitas y granitos del Cretácico (Córdova, 1988). La temperatura media anual del aire es de $25^{\circ} \mathrm{C}$ (Bullock, 1986). La precipitación promedio anual es de $780 \mathrm{~mm}$ (1983-1999), concentrándose el 80\% de las lluvias entre junio y octubre (García-Oliva et al., 1995a), debido a la influencia de los ciclones tropicales del Pacífico (García-Oliva et al., 1991). El tipo principal de vegetación es una selva baja caducifolia (Miranda y Hernández, 1963), la cual pierde la mayoría de sus hojas cuando termina la estación de lluvias (Bullock y Solís-Magallanes, 1990; Martínez-Yrízar y Sarukhán, 1990). La productividad primaria neta es de 12060 $\mathrm{kg} \mathrm{ha}^{-1} \mathrm{a}^{-1}$, de la cual el $43 \%$ es subterránea (Martínez-Yrízar et al., 1996).

La selva es transformada en praderas para uso ganadero por medio de la roza, tumba y quema. Las especies de pastos más utilizadas son guinea (Panicum maximum Jacq.) y buffel (Cenchrus ciliaris L.; GutiérrezAlcalá, 1993). Una vez transformada la selva en pradera, se deja cerca del $20 \%$ de los árboles en pie, los cuales son utilizados para sombra de ganado. Estos árboles generan islas de fertilidad con respecto al pasto (Galicia, com. pers.). La degradación del suelo se inicia desde la quema (García-Oliva et al., 1999a) y continúa durante su uso intensivo, reduciéndose principalmente la materia orgánica del suelo (García-Oliva et al., 1994) y los nutrientes (García-Oliva y Maass, 1998), debido a la erosión hídrica del suelo (Maass et al., 1988; García-Oliva et al., 1995b).

Muestreo. Las especies utilizadas fueron Caesalpinia eriostachys Benth. (Leguminosae), Cordia elaeagnoides DC. (Boraginaceae) y una especie de pasto, Panicum maximum Jacq. (Graminae). Se buscaron cinco parcelas diferentes que tuvieran: 1] más de 7 años de manejo continuo, 2] ambas especies de árboles y 3] que la distancia mínima entre los árboles escogidos en cada una de las parcelas fuera de por lo menos de 20 metros; por lo que la unidad experimental fue la parcela. En cada parcela se escogió un individuo adulto de cada una de las especies. Los árboles de C. eriostachys tuvieron una altura de 2 a $2.5 \mathrm{~m}$ y un diámetro a la altura del pecho (DAP) entre 0.38 y $0.74 \mathrm{~m} \mathrm{y}$ para C. elaeagnoides, la altura fue entre 5 y $7 \mathrm{~m}$ y el DAP entre 1.02 y $1.57 \mathrm{~m}$. En noviembre de 1996 se limitó el acceso del ganado a cada árbol y al pasto, usando alambre de púas con un cerco de $2.25 \mathrm{~m}^{2}$ de superficie. En septiembre de 1997 se colectaron muestras de $5 \mathrm{~cm}$ de profundidad asociado a las dos especies de árboles y el pasto. En el caso de los árboles, se tomaron tres muestras de suelo al azar con un nucleador de $5 \mathrm{~cm}$ de diámetro, debajo de la copa y por lo menos a $50 \mathrm{~cm}$ del tronco del árbol. Para el pasto, el cerco de exclusión se colocó donde sólo dominara Panicum maximum y que las características topográficas fueran similares a los sitios donde se encontraban los árboles en cada una de las parcelas, dentro de este cerco se tomaron tres muestras de suelo al azar en una área de $1.5 \mathrm{~m}^{2}$. En cada sitio, antes de muestrear el suelo, se removió manualmente el mantillo. Las tres muestras tomadas en la proximidad de cada individuo fueron mezcladas, transportadas al laboratorio y almacenadas a $8^{\circ} \mathrm{C}$ hasta su análisis.

Análisis de laboratorio. Las muestras se secaron en un horno a $50^{\circ} \mathrm{C}$ por 48 horas, se tamizaron con una malla de $2 \mathrm{~mm}$ y se molieron con un mortero de ágata para los siguientes análisis químicos: el carbono total (Ct) que se determinó con un analizador automatizado de $\mathrm{CO}_{2}$ UIC Mod. CM5012 y el nitrógeno total (Nt) que fue analizado con el método semi-Kjeldahl modificado para autoanalizadores (Technicon Industrial System, 1977) por medio de digestión ácida y medidos por colorimetría.

Con muestras secadas a temperatura ambiente del 
laboratorio, se determinó el pH activo (en agua desionizada con una relación 1:10, peso de suelo: volumen de agua) y el potencial (en una solución 1:5 de $0.01 \mathrm{M}$ pesado suelo: solución $0.01 \mathrm{CaCl}_{2}$ ), con un electrodo de vidrio después de agitar el suelo en la solución durante 30 minutos. La capacidad de intercambio catiónico (CIC) se midió en 1 g de suelo por medio del método de titulación EDTA (Jackson,1982).

La capacidad de la neutralización de ácidos y bases fue determinada mediante curvas de titulación propuestas por Binkley y Sollins (1990) y Rhoades y Binkley (1996). El método consiste en graficar los cambios del pH del suelo debido a la adición de ácidos y bases. Para ello, se utilizaron dos juegos de submuestras de $20 \mathrm{~g}$ de suelo secadas a temperatura ambiente dentro del laboratorio. El primer juego de submuestras se utilizó para medir la capacidad neutralizadora de ácido (ANC), se colocaron $20 \mathrm{~g}$ del suelo en una solución 2:1 (suelo:reactivo) de $1 \mathrm{M} \mathrm{KCl}$ y se agitó durante 30 minutos. Posteriormente, se le aplicaron incrementos consecutivos de $10 \mathrm{ml}$ de 0.01 $\mathrm{M} \mathrm{HCl}$ hasta que el $\mathrm{pH}$ se mantuvo constante en 4.0, mientras la muestra se mantenía en agitación continua. Se dejó reposar la muestra de 24 a 28 horas y se volvió a medir el $\mathrm{pH}$ de la solución. Este procedimiento se realizó hasta que el $\mathrm{pH}$ de la solución fuera estable en 4.0 después del tiempo de reposo. Con el segundo juego de submuestras se determinó la capacidad neutralizadora de bases (BNC): $\operatorname{los} 20 \mathrm{~g}$ de suelo se pusieron en una solución 2:1 (suelo:reactivo) de $1 \mathrm{M} \mathrm{KCl}$ y se agitó durante 30 minutos. Después, se le agregaron incrementos consecutivos de $5 \mathrm{ml}$ de $0.05 \mathrm{M} \mathrm{NaOH}$ con el mismo procedimiento que el ácido, hasta alcanzar un $\mathrm{pH}$ estable de 9.0. Antes de realizar estos procedimientos con el juego completo de las muestras, se hicieron pruebas con dos submuestras de suelo por especie, a las cuales se agregaron $0.5,1,5$ y $10 \mathrm{ml}$ de ácido y base para conocer los cambios de pH del suelo. Este ensayo nos permitió determinar la cantidad de ácido y base que fue agregado a todas las muestras. La curva de titulación se reali- zó con los valores promedio de los cinco individuos por especie. La cantidad de ácido requerida para llevar el pH a 4.0 se llamó capacidad neutralizadora del ácido $\left(\mathrm{ANC}_{4.0}\right)$ y la capacidad neutralizadora de base $\left(\mathrm{BNC}_{9.0}\right)$ fue la cantidad de base requerida para llevar el $\mathrm{pH}$ a 9.0.

El método empírico de Binkley et al. (1989) se utilizó para examinar la importancia relativa de los mecanismos (i.e. cantidad de ácido, disociación del ácido y fuerza del ácido) en determinar el $\mathrm{pH}$ del suelo. En este método, el suelo es considerado como un sistema de solución de ácido débil, el cual está afectado por 1] la cantidad de ácido, 2] el nivel de neutralización y 3] la fuerza del ácido. Este método empírico de curvas de titulación permite la interpolación secuencial de la cantidad de ácido, del nivel de neutralización y de la fuerza del ácido de un suelo a los niveles encontrados en otro suelo. Las diferencias de cada uno de los valores de $\mathrm{pH}$ interpolados con los del suelo original (el suelo de la especie que está siendo interpolado), sugieren la importancia de cada factor en el control del $\mathrm{pH}$ de este suelo. La cantidad de ácido está determinada por el tamaño del complejo de intercambio del suelo y se define como la suma de la capacidad de neutralización de ácido y base $\left(\mathrm{ANC}_{4.0}+\mathrm{BNC}_{9.0}\right)$. El grado de neutralización es la proporción del complejo de intercambio ocupado por cationes alcalinos (i.e. $\mathrm{Ca}^{2+}, \mathrm{Mg}^{2+}, \mathrm{K}^{+}$, representando la proporción de ácido disociado), relativo a los sitios que retienen cationes ácidos de $\mathrm{H}^{+} \mathrm{y} \mathrm{Al}^{3+}$ (la parte no disociada). Este grado de neutralización está definido como $\mathrm{ANC}_{4.0} /\left(\mathrm{ANC}_{4.0}+\mathrm{BNC}_{9.0}\right)$. Por último, el efecto de la fuerza del ácido se determinó como la resta entre el $\mathrm{pH}$ original menos la diferencia de los $\mathrm{pH}$ interpolados por la cantidad y por el grado de neutralización del suelo.

Para la interpolación de las curvas por la cantidad de ácido y capacidad de neutralización se utilizó la ecuación propuesta por Binkley y Sollins (1990). Lo que se interpola es el valor de ácido-base adicionado para cada valor de $\mathrm{pH}$ de la curva de titulación

Tabla.1 Promedio y error estándar $(n=5)$ del $\mathrm{pH}$ activo (en agua), $\mathrm{pH}$ potencial (en $\mathrm{CaCl}_{2}$ ), capacidad de intercambio catiónico $(\mathrm{ClC})$, concentración de carbono total $(\mathrm{Ct})$ y nitrógeno total $(\mathrm{Nt})$ del suelo asociado a dos especies de árboles remanentes y una especie de pasto. Valores con diferente letra significan diferencias entre medias a $\mathrm{p}=0.05$ con la prueba de Tukey.

\begin{tabular}{lccccc}
\hline Especie & $\mathrm{pH}\left(\mathrm{H}_{2} \mathrm{O}\right)$ & $\mathrm{PH}\left(\mathrm{CaCl}_{2}\right)$ & $\mathrm{ClC}\left(\mathrm{Meq} \mathrm{g}^{-1}\right)$ & $\mathrm{Ct}\left(\mu \mathrm{g} \mathrm{g}{ }^{-1}\right)$ & $\mathrm{Nt}\left(\mu \mathrm{g} \mathrm{g}{ }^{-1}\right)$ \\
\hline P. maximum & $6.81(0.35)$ & $6.20(0.36)$ & $31.2(6.6)$ & $32220(4,762)$ & $1946(69) \mathrm{a}$ \\
C. elaeagnoides & $7.45(0.15)$ & $6.78(0.20)$ & $42.3(5.8)$ & $31740(2,598)$ & $3664(44) \mathrm{b}$ \\
C. eriostachys & $7.13(0.24)$ & $6.66(0.24)$ & $39.7(6.6)$ & $35420(2,996)$ & $3143(314) \mathrm{b}$ \\
\hline
\end{tabular}


original de cada una de las especies. Para ello se utilizó la siguiente ecuación:

$$
(\mathrm{OH})_{{ }_{\text {nuevo }}}=(\mathrm{OH})_{\text {sp } 1} *\left((\mathrm{CA})_{\text {spl }} /(\mathrm{CA})_{\text {sp2 }}\right)
$$

donde, $(\mathrm{OH})_{\text {nuevo }}$ es la nueva coordenada de $\mathrm{X}$ que representa el valor de ácido o base en la curva interpolada, $(\mathrm{OH})_{\text {spl }}$ es la concentración de ácido o base de la curva original al $\mathrm{pH}$ que se está interpolando, $(\mathrm{CA})_{\mathrm{spl}}$ es la cantidad de ácido en la curva original y $(\mathrm{CA})_{\mathrm{sp2}}$ es la cantidad de ácido de la especie de referencia para interpolar los valores. En el caso de la interpolación por la capacidad neutralizadora, se utiliza la misma ecuación sustituyendo los valores de CA por los de la capacidad de neutralización, definida anteriormente.

Análisis estadísticos. Los análisis estadísticos se llevaron a cabo con el programa estadístico Statistix for Windows (Analytical software, 1996). Se realizaron dos tipos de análisis para poner a prueba el efecto de especie vegetal. El primero fue el análisis de varianza de una vía, donde se probó el efecto de las especies (tres especies) con cinco réplicas (parcelas), el cual se realizó en las siguientes variables: $\mathrm{Ct}, \mathrm{Nt}, \mathrm{pH}$ $\left(\mathrm{H}_{2} \mathrm{O}\right), \mathrm{pH}\left(\mathrm{CaCl}_{2}\right)$, capacidad de intercambio catiónico (CIC), capacidad neutralizadora de ácido $\left(\mathrm{ANC}_{4.0}\right)$, capacidad neutralizadora de bases $\left(\mathrm{BNC}_{9.0}\right)$ y cantidad de ácido (CA). La comparación de medias fue con el método de Diferencia Mínima Significativa de Tukey $(p=0.05)$. El segundo análisis fue un análisis de covarianza de una vía, siendo el factor principal el efecto de especie con cinco réplicas y las covariables que se utilizaron fueron la concentración de Ct y Nt del suelo, para reducir la varianza de las características de las parcelas (sitio) en el error aleatorio. Este análisis se aplicó sólo a las variables que fueron significativas en su relación con las covariables $(p<0.05)$, como en el caso del grado de neutralización (GN). La comparación de medias se hizo con el mismo método que en el análisis anterior. Las di- ferencias entre el $\mathrm{pH}$ en agua y en $\mathrm{CaCl}_{2}$ del suelo de cada una de las especies, se realizó con la prueba de $\mathrm{t}$ de Student con cinco réplicas. Para establecer correlaciones entre variable se empleó un análisis de regresión lineal para las siguientes variables: $\mathrm{Ct}$ - CIC, $\mathrm{GN}-\mathrm{pH}\left(\mathrm{H}_{2} \mathrm{O}\right), \mathrm{GN}-\mathrm{pH}\left(\mathrm{CaCl}_{2}\right)$ y $\mathrm{Ct}-\mathrm{CA}$.

\section{Resultados}

El suelo debajo de la copa de C. eriostachys presentó la mayor concentración de carbono total (Ct); sin embargo, no presentó diferencias significativas con respecto a los suelos asociados a las otras dos especies (tabla 1). En contraste, las concentraciones de nitrógeno total $(\mathrm{Nt})$ sí presentaron diferencias entre los suelos asociados a las especies $(\mathrm{p}<0.006, \mathrm{~F}=7.94$, $\mathrm{gl}=2,12$ ): en los suelos asociados a las dos especies de árboles remanentes las concentraciones fueron mayores que las del suelo asociado al pasto (tabla 1 ). La capacidad de intercambio catiónico (CIC) no mostró diferencias significativas en los suelos de las tres especies $(\mathrm{p}=0.40, \mathrm{~F}=0.97, \mathrm{gl}=2,12$ ), aunque la mayor capacidad la presentó el suelo de C. elaeagnoides y la menor el suelo de C. eriostachys (tabla 1). Se encontró una regresión débil entre CIC y $\mathrm{Ct}\left(\mathrm{r}^{2}=0.25\right.$, $\mathrm{p}=0.05$; figura 1 ).

$\mathrm{El} \mathrm{pH}$ activo (determinado en agua) del suelo de C. elaeagnoides fue 0.64 y 0.32 unidades más alto que el $\mathrm{pH}$ encontrado en los suelos de $P$. maximum y $C$. eriostachys, respectivamente, aunque las diferencias no fueron significativas $(\mathrm{p}=0.27, \mathrm{~F}=1.43$, $\mathrm{gl}=2,12$; tabla 1$)$. Las diferencias del $\mathrm{pH}$ potencial (determinado en $\mathrm{CaCl}_{2}$ ) entre los suelos de las especies fueron menores que las encontradas en el $\mathrm{pH}$ activo, pero se mantuvo la misma tendencia entre las especies: el suelo de $C$. elaeagnoides presentó el valor más alto (tabla 1). $\mathrm{El} \mathrm{pH}$ activo fue significativamente mayor que el $\mathrm{pH}$ potencial de los suelos de las tres especies: de 0.61 unidades para el pasto $(\mathrm{p}=0.003)$, de 0.67 unidades para C. elaeagnoides $(\mathrm{p}=0.002)$ y de 0.47 unidades para C. eriostachys $(\mathrm{p}<0.00013)$.

Tabla 2. Promedio y error estándar $(n=5)$ de los componentes de la acidez del suelo asociado a dos especies de árboles remanentes y una especie de pasto. $\mathrm{ANC}_{4.0}$ : capacidad neutralizadora de ácido, $\mathrm{BNC}_{9.0}$ : capacidad neutralizadora de bases, CA: cantidad de ácido y GN: grado de neutralización. Valores con diferente letra significan diferencias entre medias a $p=0.05$ con la prueba de Tukey.

\begin{tabular}{lcccc}
\hline Especie & $\mathrm{ANC}_{4.0}\left(\mathrm{~mol} \mathrm{~kg}^{-1}\right)$ & $\mathrm{BNC}_{9.0}\left(\mathrm{~mol} \mathrm{~kg}^{-1}\right)$ & $\mathrm{CA}\left(\mathrm{mol} \mathrm{kg}^{-1}\right)$ & $\mathrm{GN}(\%)$ \\
\hline P. maximum & $-0.0404(0.001) \mathrm{b}$ & $0.0680(0.009) \mathrm{b}$ & $0.1115(0.013) \mathrm{b}$ & $0.3353(6.618) \mathrm{b}$ \\
C. elaeagnoides & $-0.1515(0.002) \mathrm{a}$ & $0.0768(0.008) \mathrm{b}$ & $0.1728(0.020) \mathrm{ab}$ & $0.7023(5.897) \mathrm{a}$ \\
C. eriostachys & $-0.0909(0.002) \mathrm{a}$ & $0.1434(0.024) \mathrm{a}$ & $0.2493(0.027) \mathrm{a}$ & $0.4104(6.618) \mathrm{b}$ \\
\hline
\end{tabular}


La capacidad neutralizadora del ácido $\left(\mathrm{ANC}_{4.0}\right)$ del suelo de P. maximum fue $73 \%$ menor que la del suelo de $C$. elaeagnoides y $55 \%$ menor que el suelo de $C$. eriostachys ( $\mathrm{p}<0.02, \mathrm{~F}=5.68, \mathrm{gl}=2,12$; Tabla 2$)$. De igual manera, el suelo de $P$. maximum tuvo una capacidad neutralizadora de la base $\left(\mathrm{BNC}_{9.0}\right) 52 \%$ menor que la del suelo de $C$. eriostachys y fue parecida a la del suelo de $C$. elaeagnoides ( $\mathrm{p}<0.01, \mathrm{~F}=6.77$, gl=2,12; tabla 2 ).

La suma absoluta de $\mathrm{ANC}_{4.0}$ y $\mathrm{BNC}_{9,0}$ define a la cantidad de ácido (CA), que fue la cantidad de $\mathrm{H}^{+} \mathrm{y}$ $\mathrm{OH}^{-}$requerida para que los suelos cambiaran el $\mathrm{pH}$ entre 4.0 y 9.0. Se encontraron diferencias de CA entre el suelo de las especies $(\mathrm{p}=0.004, \mathrm{~F}=8.05, \mathrm{gl}=2,12)$, donde el suelo de P. maximum tuvo un valor $55 \%$ menor que el valor del suelo de $C$. eriostachys (tabla 2). De todo lo anterior, se puede concluir que el suelo asociado a $P$. maximum tiene la menor capacidad buffer a los cambios de $\mathrm{pH}$ (el valor menor de CA), mientras que el suelo de C. elaeagnoides neutraliza mejor los ácidos (el valor más negativo de $\mathrm{ANC}_{4.0}$ ) y el suelo de $C$. eriostachys tiene mayor capacidad de neutralización de bases (el valor mayor de $\mathrm{BNC}_{9,0}$, tabla 2 y figura 2).

Así mismo, el efecto de las especies también fue significativo $(\mathrm{p}<0.001, \mathrm{~F}=12.54$, gl $=2,10)$ con el grado de neutralización (GN): el suelo de C. elaeagnoides presentó un valor mayor que el suelo de las otras dos especies (tabla 2), lo cual significa que el $70 \%$ de los sitios del complejo de intercambio catiónico está siendo ocupado por cationes alcalinos. En el análisis de covarianza aplicado, el GN se correlacionó significativamente con $\mathrm{Ct}(\mathrm{p}<0.003)$ y Nt $(\mathrm{p}=0.005)$. $\mathrm{La}$ regresión del $\mathrm{pH}$ activo con $\mathrm{GN}$ fue mayor $\left(\mathrm{r}^{2}=0.64\right.$, $\mathrm{p}=0.0003)$ que la de $\mathrm{GN}$ y el $\mathrm{pH}$ potencial $\left(\mathrm{r}^{2}=0.57\right.$, $\mathrm{p}=0.001$ ).

Utilizando el método empírico de interpolación de las curvas de titulación de un suelo a otro, fue posible determinar el peso de cada uno de los factores en el pH del suelo. En la tabla 3 se presentan cuatro ejemplos de los valores del $\mathrm{pH}$ original y sus interpolaciones utilizando otra especie para cada uno de los tres factores considerados: cantidad del ácido, grado de neutralización y fuerza del ácido. Por ejemplo, cuando se interpolaron los valores de $\mathrm{pH}$ del suelo de $P$. maximum con la curva del suelo de $C$. elaeagnoides, el $\mathrm{pH}_{\text {original }}$ (5.69) presentó la mayor diferencia con el $\mathrm{pH}$ interpolado por la fuerza del ácido (FA, $\mathrm{pH}_{\text {interpolado }}$ 4.20 ), por lo que la fuerza del ácido fue el factor con mayor peso para el suelo de $P$. maximum. Cuando se interpolaron los valores de $\mathrm{pH}$ del suelo de las dos especies de los árboles a la curva del suelo de $P$. maximum, el factor principal fue la CA, ya que los valores de $\mathrm{pH}$ interpolados por la CA presentaron la mayor diferencia con el valor de $\mathrm{pH}_{\text {original }}$ correspondiente: 0.31 unidades para el suelo de $C$. eriostachys y 0.75 unidades para el suelo de $C$. elaeagnoides. Cuan-

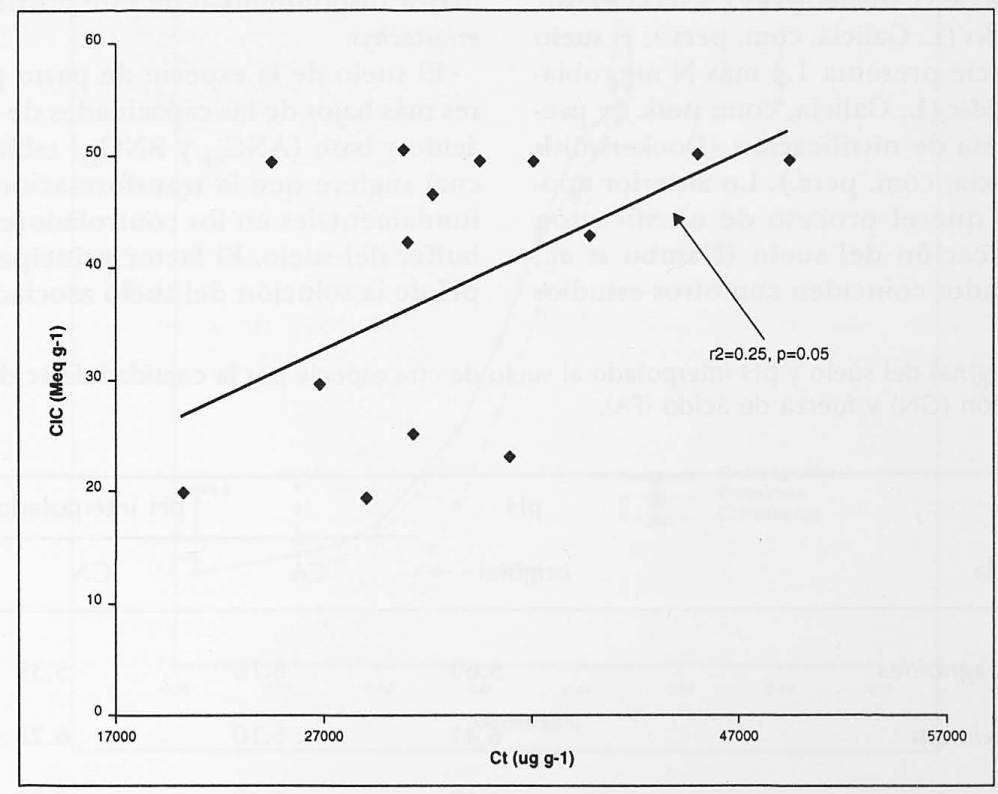

Figura 1. Regresión lineal entre el Carbono total (Ct) y la capacidad de intercambio catiónico (CIC) del suelo de dos especies de árboles remanentes y una especie de pasto $(n=15)$. 
do se interpolaron los valores de $\mathrm{pH}$ del suelo entre las dos especies de árboles remanentes, la CA volvió a ser el factor más importante.

\section{Discusión}

Los resultados del presente trabajo mostraron que la identidad de la especie vegetal es muy importante en los mecanismos que controlan el $\mathrm{pH}$ del suelo. La cantidad de ácido (CA) fue el factor principal que controla la dinámica del $\mathrm{pH}$ del suelo de ambas especies de los árboles remanentes de la selva, el cual está afectada por la identidad de la especie del árbol. La diferencia fundamental entre el suelo de ambas especies fue el grado de neutralización (GN, tabla 2), ya que el complejo de intercambio catiónico del suelo de $C$. elaeagnoides estuvo ocupado principalmente por los cationes alcalinos a diferencia del suelo de C. eriostachys, que estuvo ocupado principalmente por $\mathrm{H}^{+}$, lo cual mantuvo más ácida la solución del suelo asociado a esta última especie. Este efecto también se expresó en la diferencia entre el $\mathrm{pH}$ activo (en agua) y el $\mathrm{pH}$ potencial (en $\mathrm{CaCl}_{2}$ ), siendo mayor en el suelo de C. elaeagnoides que el de C. eriostachys $(0.67 \mathrm{y}$ 0.47 unidades, respectivamente). Las causas que pueden explicar esta diferencia están relacionadas con el ciclaje del nitrógeno, ya que existe evidencia de que C. eriostachys tiene mayor $\mathrm{N}$ disponible en el suelo (Dockersmith et al., 1999). Por ejemplo, la concentración de $\mathrm{N}$ en la hojarasca de $C$. eriostachys es 1.4 veces mayor que en C. elaeagnoides (L. Galicia, com. pers.), el suelo de la primera especie presenta 1.4 más $\mathrm{N}$ microbiano que C. elaeagnoides (L. Galicia, com. pers.) y presenta una mayor tasa de nitrificación (Dockersmith et al., 1999; L. Galicia, com. pers.). Lo anterior apoya la hipótesis de que el proceso de nitrificación aumenta la acidificación del suelo (Nambu et al., 1994). Estos resultados coinciden con otros estudios con especies fijadoras de $\mathrm{N}$ en plantaciones de ecosistemas templados (Binkley y Sollins, 1990) y de ecosistemas tropicales (Rhoades y Binkley, 1996).

Así mismo, la CA depende de la capacidad de adsorción que tiene el complejo de intercambio catiónico y aniónico del suelo, el cual está formado tanto por humus como por arcillas. En el sitio de estudio, la arcilla dominante es la caolinita (Campo, 1995), la cual se caracteriza por poca capacidad de intercambio catiónico (Buckman y Brady, 1970), por lo que probablemente el complejo de intercambio catiónico depende principalmente del humus, lo cual explica la correlación entre la CA con el Ct $\left(\mathrm{r}^{2}=0.46, \mathrm{p}=0.01\right)$. La cantidad y calidad del humus de suelo están afectadas por las especies vegetales a través de su productividad y la composición química del material vegetal que retornan al suelo, modificando a su vez la tasa de descomposición del mismo (García-Montiel y Binkley, 1998).

Las diferencias del $\mathrm{pH}$ de la solución del suelo asociado a ambas especies generan cambios en el ambiente fisico-químico del mismo, afectando: 1] la presencia de grupos microbianos, tales como los hongos que prefieren condiciones de neutras a ácidas (Prasad et al., 1994) y 2] el equilibrio químico de la solución del suelo, modificando los procesos de disponibilidad de los nutrientes; por ejemplo, la mayor disponibilidad de fósforo se presenta en $\mathrm{pH}$ entre 6 y 7 (Agbenin y Tiessen, 1994), por lo que se espera mayor disponibilidad de este nutriente debajo de $C$. eriostachys.

El suelo de la especie de pasto presentó los valores más bajos de las capacidades de neutralización de ácido y base $\left(\mathrm{ANC}_{4.0}\right.$ y $\mathrm{BNC}_{9.0}$, tabla 2 y figura 2$)$, lo cual sugiere que la transformación genera cambios fundamentales en los controladores de la capacidad buffer del suelo. El factor principal que controló el $\mathrm{pH}$ de la solución del suelo asociado al pasto, fue la

Tabla 3. $\mathrm{pH}$ original del suelo y $\mathrm{pH}$ interpolado al suelo de otra especie por la cantidad de ácido (CA), grado de neutralización (GN) y fuerza de ácido (FA).

\begin{tabular}{lrrrr}
\hline Especie & $\mathrm{pH}$ & \multicolumn{3}{c}{$\mathrm{pH}$ interpolado } \\
\cline { 4 - 5 } Original - interpolada & original & $\mathrm{CA}$ & $\mathrm{GN}$ & $\mathrm{FA}$ \\
\hline P. maximum- C. elaeagnoides & 5.69 & 6.10 & 5.39 & 4.20 \\
C. eriostachys-P. maximum & 6.41 & 6.10 & 6.25 & 6.40 \\
C. elaeagnoides- P. maximum & 6.15 & 5.40 & 5.50 & 5.50 \\
C. elaeagnoides- C. eriostachys & 6.15 & 6.80 & 6.20 & 6.20 \\
\hline
\end{tabular}


fuerza del ácido (FA), la cual depende de la naturaleza química de los ácidos presentes en la solución. Esto último es crítico en las condiciones de la pradera, porque las quemas recurrentes generan cambios drásticos en la naturaleza de los ácidos que se están incorporando al suelo y por lo tanto, llevan a transformaciones radicales en el equilibrio químico de la solución.

Las consecuencias de la reducción de la capacidad buffer en el suelo asociado al pasto, generan un desequilibrio químico de la solución del suelo, por lo que se incrementa la velocidad del ciclaje de nutrientes, trayendo consigo una mayor pérdida por lixiviación (Singh, 1989) y una mayor susceptibilidad del suelo a la degradación por las quemas continuas, las cuales reducen a la materia orgánica del suelo y la actividad microbiana (García-Oliva et al., 1999b). Por lo anterior la recuperación de los controladores del $\mathrm{pH}$ es uno de los retos más importantes a vencer en la restauración de estos suelos, donde la interacción entre el suelo y las especies vegetales es un elemento fundamental.

\section{Agradecimientos}

A dos revisores anónimos y a Sonia A. Álvarez-Santiago por sus comentarios al manuscrito, a Georgina
García-Méndez por su apoyo técnico en el laboratorio, a Heberto Ferreira por su apoyo técnico en el análisis de datos, al Sr. Ramiro Peña por permitirnos usar sus parcelas. Al personal de la Estación de Biología Chamela por todo el apoyo logístico durante el trabajo de campo. Este proyecto se realizó con el financiamiento de DGAPA-UNAM.

\section{Literatura citada}

Agbenin J.O. y Tiessen H. 1994. Phosphorus transformations in a toposequence of Lithosols and Cambisols from semi-arid northeastern Brazil. Geoderma 62: 345-362.

Alban D.H. 1982. Effects of nutrient accumulation by aspen, spruce, and pine on soil properties. Soil Science Society American Journal 46:853-861.

Analytical Software 1996. Statistix for windows, User's manual. Analytical Software. Tallahassee, FL., USA.

Binkley D. Valentine D., Wells C. and Valentine U. 1989. An empirical analyses of the factors contributing to 20year decrease in soil $\mathrm{pH}$ in an old-field plantation of loblolly pine. Biogeochemistry 7:39-54.

Binkley D. and Hart S.C. 1989. The Components of Nitrogen availability assessment in forest soils. Advances in Soil Sciences 10:57-112.

Binkley D. and Sollins P. 1990. Factors determining differences in soil $\mathrm{pH}$ in adjacent conifer and Alder-Conifer

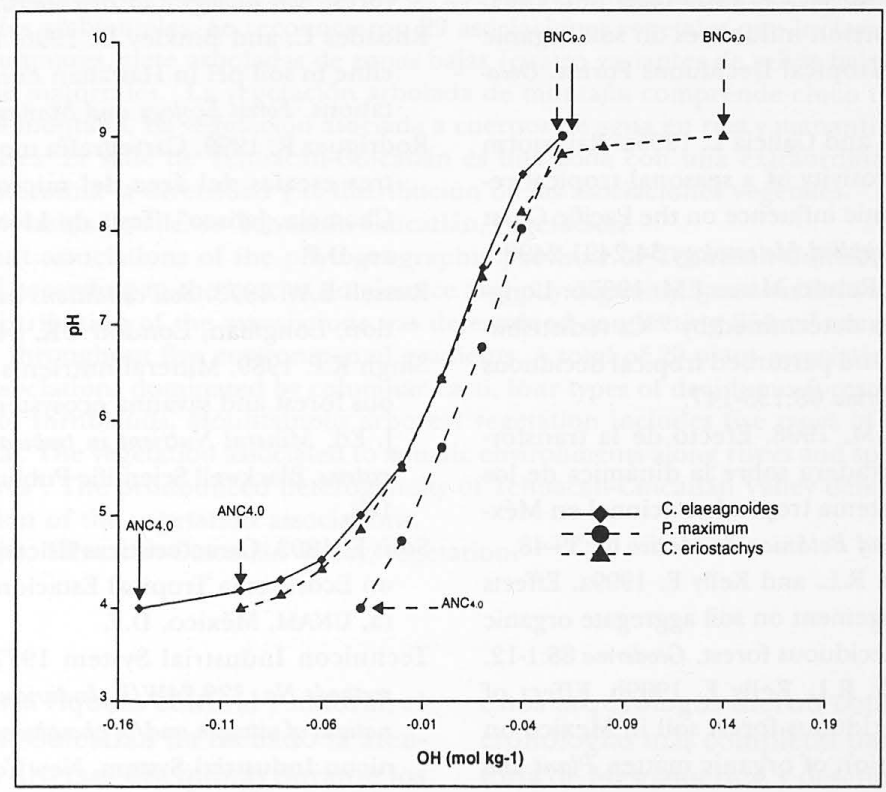

Figura 2. Curvas de titulación para suelos asociados a dos especies de árboles remanentes (C. elaeagnoides y $C$. eriostachys) y a la especie de pasto $\left(P\right.$. maximum). $\mathrm{ANC}_{4.0}$ : capacidad neutralizadora de ácido, $\mathrm{BNC}_{9.0}$ : capacidad neutralizadora de bases. 
Stands. Soil Science American Journal 54:1427-1433.

Buckman H.O. y Brady N.C. 1970. Naturaleza y propiedad de los suelos. UTEHA, México, D.F.

Bullock S.H. 1986. Climate of Chamela, Jalisco and trends in the south coastal region of Mexico. Archives for Meteorology, Geophysics, and Bioclimatology. 36:297-316.

Bullock S.H. y Solís-Magallanes A. 1990. Phenology of canopy trees of a tropical deciduous forest in Mexico. Biotropica 21:22-35.

Campo J. 1995. Ciclo del fósforo en un ecosistema tropical estacional. Tesis doctoral, UNAM, México, D.F.

Córdova C. 1988. Cartografía geomorfológica en escala pequeña del occidente de la República Mexicana. Tesis de Licenciatura, UNAM, México, D.F.

Dockersmith I.C., Giardina C.P. and Sanford Jr. R.L. 1999. Persistence of tree related patterns in soil nutrients following slash-and-burn disturbance in the tropics. Plant and Soil 209:137-156.

Finzi A.C., Canham C.D. and van Breemen N. 1998. Canopy tree-soil interactions within temperate forests: species effects on $\mathrm{pH}$ and cations. Ecological Applications 8:447-454.

García-Montiel D. and Binkley D. 1998. Effect of Eucalyptus saligna and Albizia falcataria on soil processes and nitrogen supply in Hawaii. Oecologia 113:547-556.

García-Oliva F., Ezcurra E. and Galicia L. 1991. Pattern of rainfall distribution in the Central Pacific coast of Mexico. Geografiska Annaler 73A:179-186.

García-Oliva F., Casar I., Morales P. and Maass J.M. 1994. Forest-to-pasture conversion influences on soil organic carbon dynamics in a Tropical Deciduous Forest. Oecologia 99:392-396.

García-Oliva F., Maass J.M. and Galicia L. 1995a. Rainstorm analysis and rainfall erosivity of a seasonal tropical region with a strong cyclonic influence on the Pacific Coast of Mexico. Journal of Applied Meteorology 34:2491-2498.

García-Oliva F., Martínez R. and Maass J.M. 1995b. Longterm net soil erosion as determined by ${ }^{137} \mathrm{Cs}$ redistribution in an undisturbed and perturbed tropical deciduous forest ecosystem. Geoderma 68:135-147.

García-Oliva F. y Maass J.M. 1998. Efecto de la transformación de la selva a pradera sobre la dinámica de los nutrientes en un ecosistema tropical estacional en México. Boletín de la Sociedad Botánica de México 62:39-48.

García-Oliva, F. Sanford Jr. R.L. and Kelly E. 1999a. Effects of slash-and-burn management on soil aggregate organic $\mathrm{C}$ and $\mathrm{N}$ in a tropical deciduous forest. Geoderma 88:1-12.

García-Oliva F. Sanford Jr. R.L. Kelly E. 1999b. Effect of burning of tropical deciduous forest soil in Mexico on the microbial degradation of organic matter. Plant and Soil 206: 29-36.

Gutiérrez-Alcalá A.R. 1993. La ganadería extensiva en el trópico seco mexicano: causas, consecuencias y manifestaciones en su medio social. Tesis de Licenciatura,
UNAM, México, D.F.

Howard P.J.A., Howard D.M. and Lowe L.E. 1998. Effects of tree species and soil physico-chemical conditions on the nature of soil organic matter. Soil Biology and Biochemistry 30:285-297.

Jackson M.L. 1982. Análisis quimicos de suelos. De. Omega, Barcelona.

Jenny H. 1941. Factors of soil formation. McGraw-Hill, New York.

Maass J.M., Jordan C. and Sarukhán J. 1988. Soil erosion and nutrient losses in a seasonal tropical agroecosystems under various management techniques. Journal Applied of Ecology 25:595-607.

Martínez-Yrízar A. and Sarukhán J. 1990. Literfall patterns in a tropical deciduous forest in Mexico over a five-year period. Journal of Tropical Ecology 6: 433-444.

Martínez-Yrízar A., Maass J.M., Pérez-Jiménez L.A. and Sarukhán J. 1996. Net primary productivity of a tropical deciduous forest ecosystem in western Mexico. Journal of Tropical Ecology 12:169-175.

Miranda F. y Hernández X. E. 1963. Los tipos de vegetación en México y su clasificación. Boletín de la Sociedad Botánica de México 28:29-179.

Nambu K., Kummatsu T. and Kyuma K. 1994. Rates of soil acidification under different patterns of nitrogen mineralization. Soil Science Plant Nutrition 40:95-104.

Prasad P. Basu S. y Behera N. 1994. A comparative account of the microbiological characteristics in a dry tropical forest Soil Biology and Biochemistry 24:145-150.

Rhoades C. and Binkley D. 1996. Factors influencing decline in soil pH in Hawaiian Eucalyptus and Albizia plantations. Forest Ecology and Management 80: 47-56.

Rodríguez R. 1999. Cartografía morfométrica jerárquica a tres escalas del área del microbloque "El Colorado" Chamela, Jalisco". Tesis de Licenciatura, UNAM, México, D.F.

Russell E.W. 1973. Soil conditions and plant growth. $10^{\text {th }}$ edition, Longman, London UK, 849 pp.

Singh K.P. 1989. Mineral nutrients in tropical dry deciduous forest and savanna ecosystems in India. In: Proctor J. Ed. Mineral Nutrient in tropical forest and savanna ecosystems. Blackwell Scientific Publications, Cambridge, 153168.

Solís E. 1993. Características fisicoquímicas de un suelo en un Ecosistema Tropical Estacional. Tesis de Licenciatura, UNAM, México, D.F.

Technicon Industrial System 1977. Technicon publication methods No. 329-74W/B. Individual /simultaneous determinations of nitrogen and/or phosphorous in $B D$ acid digest. Technicon Industrial System, New York.

van Miegroet H. and Cole D.W. 1984. The impact of nitrification on soil acidification and cation leaching in a red alder ecosystem. Journal of Environmental Quality 13:586-590. 